\title{
The process of protecting metals from corrosion
}

\author{
Smirnova Zhanna V. ${ }^{1}$, Vaganova O.I ${ }^{2}$., Cherney O.T. ${ }^{3}$, Mukhina M.V ${ }^{4}$, Kutepova L.I. ${ }^{5}$ \\ ${ }^{1}$ Minin Nizhny Novgorod State Pedagogical University (Minin University), Nizhny Novgorod, Russian \\ Federation, vaganova_o@mail.ru \\ ${ }^{2}$ Minin Nizhny Novgorod State Pedagogical University, Nizhny Novgorod, Russian Federation \\ z.v.smirnova@mininuniver.ru \\ ${ }^{3}$ Minin Nizhny Novgorod State Pedagogical University (Minin University), Nizhny Novgorod, Russian \\ Federation, fiolet1975@mail.ru \\ ${ }^{4}$ Minin Nizhny Novgorod State Pedagogical University (Minin University), Nizhny Novgorod, Russian \\ Federation, mariyamuhina@yandex.ru \\ ${ }^{5}$ Minin Nizhny Novgorod State Pedagogical University (Minin University), Nizhny Novgorod, Russian \\ Federation, lubovkutepova@mail.ru
}

\begin{abstract}
One of the problems of industrial production and construction is the destruction of metal structures. Many scientists and specialists in the field of chemical research deal with this problem to preserve the metal properties of structures for a longer period. To maintain the health of metal and alloys, there are several processing methods and chemical compounds to protect the structure from corrosion. This article discusses methods and methods of protecting metal structures and alloys.
\end{abstract}

Key words: corrosion, metal, chemical properties, electrochemical corrosion.

\section{INTRODUCTION}

Metalwork, like all living things on earth, have the property of diseases, such properties include surface wear, deformation of the parts and the most dangerous wear of metal structures this is metal corrosion.

All metals in their natural state are in the form of chemical compounds with oxygen, sulfur and many other chemical elements: $\mathrm{Fe} 3 \mathrm{O} 4, \mathrm{Fe} 2 \mathrm{O} 3, \mathrm{FeO}, \mathrm{Al} 2 \mathrm{O} 3$, etc. Pure metal alloys are obtained by the method of extracting metal particles from these oxides. Subsequently, machine parts, metal structures, etc. are made from the extracted metal. Natural phenomena, in turn, return their processes to their circles - metal oxidation occurs, as a result of which it is necessary to re-conduct the chemical process of extracting metal particles [1].

In the entire history of the existence of mankind, about 43 billion tons of iron have been mined, about 6 billion tons. It is now in cars and building structures, and 16 billion tons. already eaten by rust, i.e. became unusable. A lot of time and labor are spent on repairing corroded machines and building structures, on replacing water pipes, heating pipes, on corrosion protection and corrosion prevention. As a result, about $40 \%$ of the metal produced annually is spent on restoring corrosion losses.

\section{2. . LITERATURE REVIEW}

There are two types of corrosion: electrochemical and chemical.

Three types of factors Figure 1 determine the corrosion process.

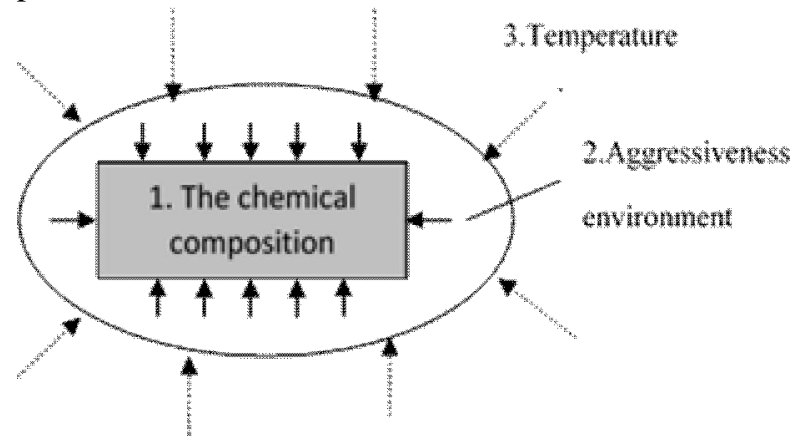

Figure 1: Three groups of factors affecting corrosion of products

By definition, chemical corrosion refers to the destruction of metals after exposure to high temperature gases or liquids.

At high temperatures, carbon deposits form on valves and cylinder heads, on spark plugs, on pistons (top of the skirt and piston grooves), on compression rings, on furnace fittings, and on heat and electric heaters. First, varnish coatings are formed on the surfaces of parts at temperatures up to $150^{\circ} \mathrm{C}$, then carbon deposits appear at higher temperatures [2].

The process of electrochemical corrosion occurs in liquid media, which conducts an electric current in the process, electrolytic destruction of the metal occurs. 
Electrochemical corrosion takes place under certain conditions: only in the presence of water. Water is in the atmosphere, in the soil, on the surfaces of parts and structures. Soils, air and working media also contain oxides and salt crystals, sulfur and exhaust gases, and, as a result, acids are formed - this is the second condition for the appearance of corrosion.

\section{RESULT AND DISCUSSION}

In the process of research, we examined the process of metal corrosion using the example of the presence of zinc and copper in the structure.

According to the theory, in an acidic medium all zinc atoms leave their electrons on the anode, as a result of which they turn into positively charged zinc ions, passing into a liquid. At the cathode, copper plates, hydrogen anions receive electrons, thus, hydrogen atoms from the solution will be released at the cathode.

According to this theoretical study, we see that the process of electrochemical corrosion is possible under the following conditions:

- the presence of water;

- the presence of acids and salts;

- the presence of different metals.

The metal atoms in contact with the electrolyte pass into the solution in the form of ions. The transition of atoms into ions is determined by the magnitude of the normal electronic potential (Figure 2).

The electronic potential is the voltage $(\mathrm{V})$ of the electric current that must be applied to the metal-liquid phase interface to prevent the metal ion from passing into the solution.

The larger the negative potential value (-), the more the metal tends to dissolve in electrolytes. the more intense the corrosion.

Metals with a negative hydrogen potential (-) displace hydrogen from acids, and metals with a positive potential $(+)$ are displaced by hydrogen from a solution [3].

In the case of a thick dense film, corrosion stabilization is possible. Such films are formed on aluminum, lead, tin, nickel and chromium. They do not pass gases to the surface of the part. Dense films are also created on the iron, but they crack and therefore peel off from the iron. Technical iron rusts at ordinary temperatures, at higher temperatures $(250 \ldots$ $300{ }^{\circ} \mathrm{C}$ ) a rust film is formed right before the eyes, and at 600 ${ }^{0} \mathrm{C}$ the surface of iron is very quickly covered with a thick layer of scale. Due to the fact that the film is porous, gases penetrate well to the metal surface and the corrosion rate does not decrease, because no protective effect from the film.

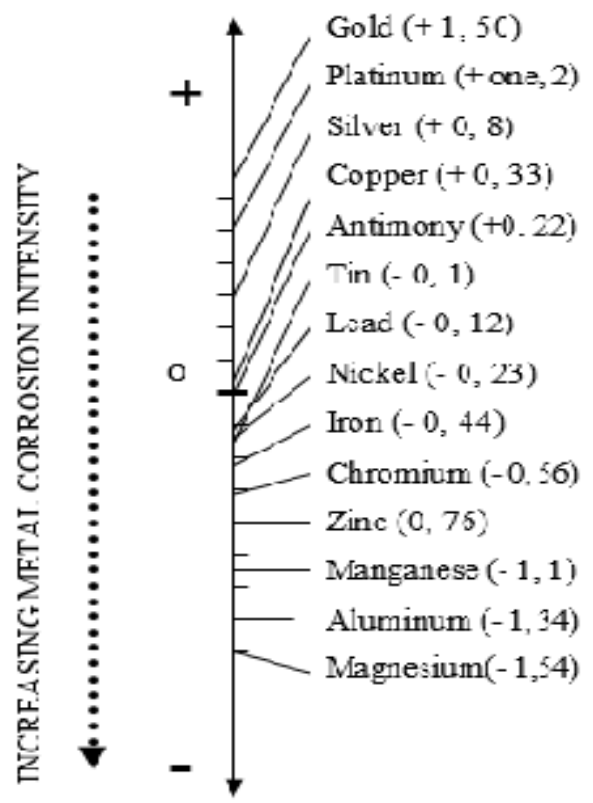

Figure 2: Hydrogen potentials of metals.

From Figure 3, we see that the corrosion damage can be different: solid, local and intercrystalline.

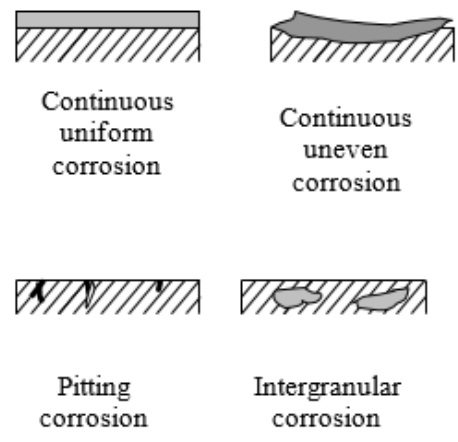

Figure 3: Types of Corrosion Damage

According to Figure 3, we see that corrosion decreases with the passage of the following measures:

- the correct selection of material details;

- decrease in the aggressiveness of the environment;

- creation of a protective layer.

Figure 4 shows the methods of corrosion protection, which are determined by the need to neutralize factors, factors are determined by the intensity of corrosion, i.e. the correct selection of anticorrosive materials, lowering the temperature of the medium, preventing the surface from aggressive environment [4].

Consider one of the factors for reducing corrosion.

The most common method is the selection of metal. The metal selection process is shown in Figure 2, we see that products made of gold, platinum and silver are more corrosion resistant than products made of iron, zinc and aluminum, but these materials are expensive, therefore, they are of limited use in everyday life and technology. 
Aluminum and its alloys have a very low hydrogen potential, but strong and resistant protective films form relatively quickly on the surface of the part, so aluminum parts do not require additional protection (painting, etc.) from corrosion.

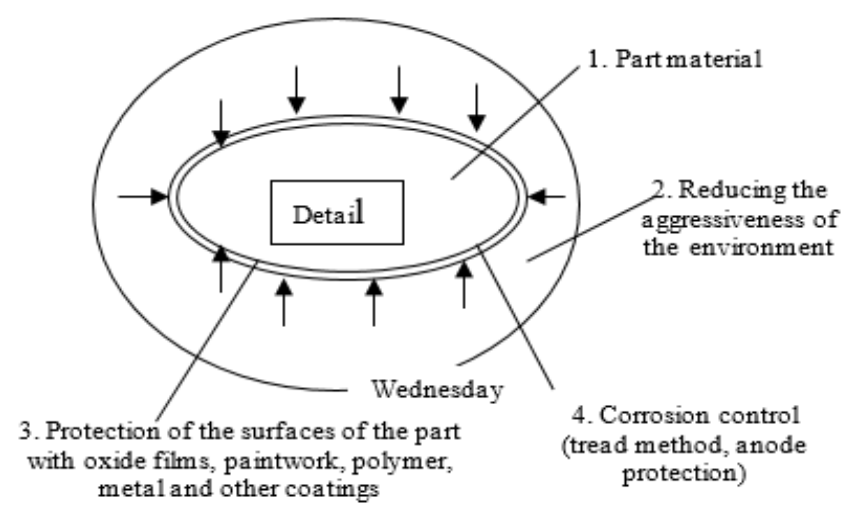

Figure 4: Methods for protecting parts from corrosion.

The aggressive environment is reduced by the addition of antioxidant additives to lubricating oil and fuel in cooling systems. For example, in the heating system, important factors are the decrease in the temperature of the coolant for heating systems from $100 \ldots 1100 \mathrm{C}$ to $60 \ldots 70$ 0C, which, of course, will lead not only to a reduction in heat loss, but also to a decrease in corrosion of system elements.

There is another method of reducing corrosion - this is coating the surface with various finishing materials, preventing the process of electrochemical corrosion. The coatings themselves are applied to the surface of the part and structure [5].

The method of protecting metals with protectors is shown in Figure 5 is that metal plates are connected to the pipeline through a cable, having a lower hydrogen potential, which,

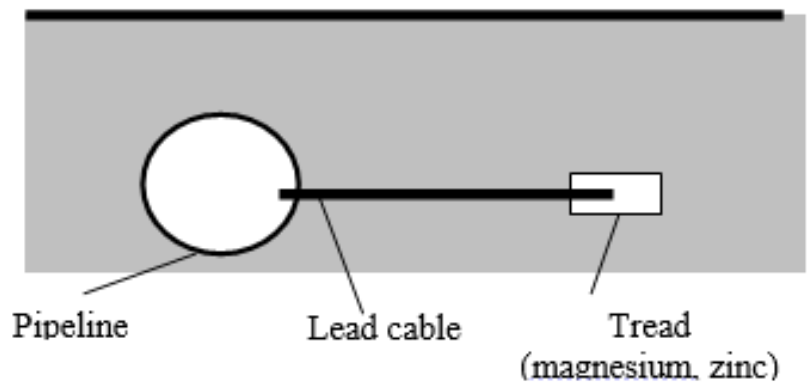

Figure 5: Scheme of protection of metals from corrosion by protectors.

of course, dissolves and collapses in the first place, protecting the main product from corrosion.

In Figure 6, another method is investigated - protection by external current-metal structures. This method is common among the manufacture of ship structures, trunk pipelines and the manufacture of underground building structures.

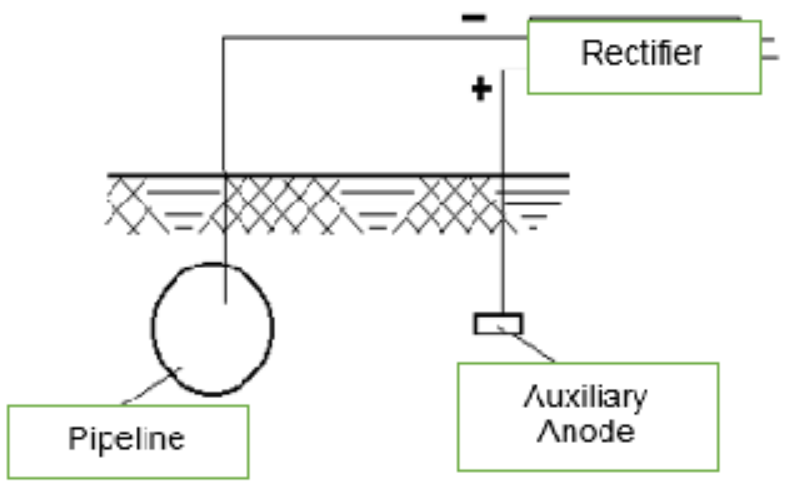

Figure 6: Cathode Protection pipelines against corrosion.

This method differs from the method of protectors in that a negative potential from the power source is supplied to the protected part, and a positive potential to the auxiliary anode. The technological way to protect the structure from corrosion is painting. Figure 7 shows the process of protecting the metal when painting the surface.

There are the following methods for surface treatment against corrosion: puttying, phosphating and primer.

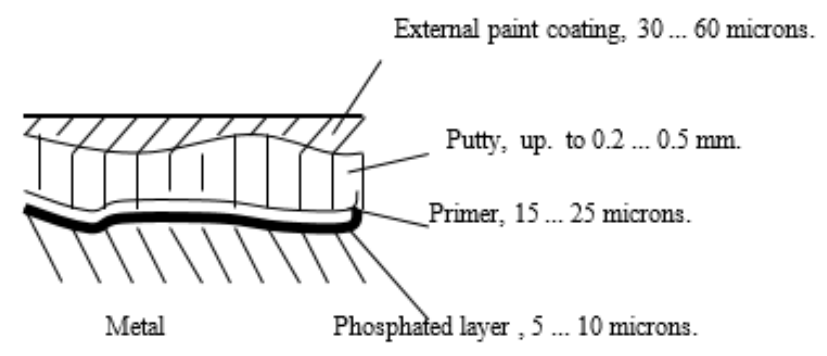

Figure 7: Paintwork scheme of the part.

Phosphating the surface is obtained by treating parts with phosphoric acid or a salt solution, or phosphating soil.

A primer is necessary to ensure good adhesion of the metal to paint or putty and to protect the metal from corrosion.

Puttying the surface is necessary to level the surface from scratches, scratches, defects in welds, and also to protect the metal from corrosion.

For painting, nitro enamels and synthetic enamels are used. The advantage of nitroenamels is drying at natural temperature for $10 \ldots 15$ minutes. In total, $5 \ldots 6$ layers of a thickness of $8 \ldots 10$ microns are applied with the obligatory drying of each layer. However, the nitro-enamel coating has insufficient corrosion resistance and requires complex technological polishing. The surface is first sanded and coated with a solvent, then polished with pastes.

Synthetic enamels give the coating a more durable gloss and better protection against corrosion. Their service life is $4 . .5$ years. The number of layers is enough $2 \ldots 3$ and polishing is greatly simplified. However, the polymerization of a synthetic coating (drying) occurs at a high temperature of $120 \ldots 130^{\circ} \mathrm{C}$ for an hour, which requires the use of sophisticated equipment. 
Smirnova Zhanna V et al., International Journal of Emerging Trends in Engineering Research, 8(5), May 2020, 1728 - 1731

Paintwork can be applied in the following ways:

1 - manual painting with a brush;

2 - painting by immersion of the part in a bath with paint;

3 - application of paint by air spray;

4 - application of paint by airless spraying;

5- coloring in an electrostatic field (Fig. 8).

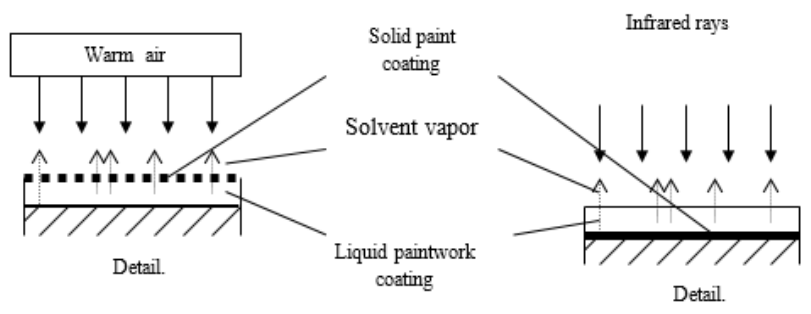

Figure 8: Scheme of convection (warm air) and thermal radiation (infrared rays) drying coatings.

The most effective technology is painting in an electrostatic field (Fig. 8). However, preliminary painting of the inner surfaces and deep depressions is necessary. In addition, part of the paint, without receiving a charge, is lost. Almost 100\% of paint use is obtained by electromechanical spraying, when a negative charge of high voltage is communicated not to the intermediate medium (air), but directly to the paint .During thermoradiation drying, the heat source is invisible infrared rays, which freely pass through the coating layer and heat the metal. The evaporation of the solvent and the formation of a crust begins from the bottom of the layer, thereby creating good conditions for the removal of vapors. The polymerization process is also faster. Thermo-radiation drying is almost twice as fast as convection drying.

As sources of infrared radiation, heat emitters are used, heated by electric current or gas. Panels of emitters are heated to $400 \ldots 500{ }^{\circ} \mathrm{C}$ and emit infrared rays with a wavelength of 3 ... 5 microns, which easily pass the paint layer and, being absorbed by the metal, heat it.

The "usefulness" of rust. First of all, the benefits of corrosion are a return to their circles. Pipes, sheet, channel and other metal buried as a result of careless work in the ground, recessed into swamps and rivers, returns to its natural state oxides (ore)

\section{CONCLUSION}

Summing up the effects of metal processing on corrosion, I would like to note: Architect John Dinel created a "rusty building" in Moline in 1959, believing that people should be sick of looking at polished aluminum facades and humanity should return to a more rude style. The Institute of American Architecture awarded the "rusty" building a gold medal. In
Chicago, the Civin Center skyscraper was built, which is covered in rust in rain [6].

The bridge over the Consumnes River, a few months after construction, acquired a beautiful and rare greenish-brown color (such low-carbon steel was selected that it was coated with such a film under atmospheric conditions).

There are bridge steels that rust for $2 \ldots 4$ years, and then a black and dense film is formed that adheres tightly to the metal and reliably protects it from further corrosion

\section{REFERENCES}

1. Smirnova Zh.V., Mukhina M.V., Katkova O.V., Gruzdeva M.L., Chernei O.T. Network interaction as a factor of professional qualities 'development of service workers // Lecture Notes in Networks and Systems (see books). 2020.Vol. 87.S. 698-704.

https://doi.org/10.1007/978-3-030-29586-8_81

2. Bartels, N. A. Metallography and heat treatment of metals / N.A. Bartels. - M.: State Scientific and Technical Publishing House, 2018. - 376 c.

3. Barkhatov N.A., Revunov S.E., Mukhina M.V., Gruzdeva M.L., Cherney O.T., Smirnova Z.V. Establishing the orientation of shock wave plane of solar wind magnetic cloud for conclusions about the level of auroral substorm activity JP Journal of Heat and Mass Transfer. 2019. Vol. 17. No. 1. S. 195-202.

https://doi.org/10.17654/HM017010195

4. Smirnova Zh.V., Cherney O.T. Technology of construction materials study guide / University of Minin. Nizhny Novgorod, 2019.

5. Y. Pratapa Reddy, Dr. K. L Narayana, Dr. M. Kedar Mallik.

Electro-chemical behavior of different metals in Sodium Chloride solution.// International Journal of Emerging Trends in Engineering Research, 7(11), November 2019, 71Volume 7, No. 11 November 2019.

6. Eko Julianto, Waluyo Adi Siswanto, Marwan Effendy Characteristics of Temperature changes and Stress of Float Glass under Heat Radiation// International Journal of Emerging Trends in Engineering Research, Volume 7, No. 9 September 2019.

https://doi.org/10.30534/ijeter/2019/03792019 